\title{
LAS PERIOdistas DESDE LOS ESTUdIOS DEL PERIODISMO: PERFILES PROFESIONALES DE LAS MUJERES EN LOS MEDIOS INFORMATIVOS
}

\section{Female journalists from journalism studies: professional profiles of women in Media}

\author{
Adriana Amado \\ adrianacatedraa@gmail.com \\ Universidad de Castilla-La Mancha - España
}

Recibido: 28-02-2017

Aceptado: 02-05-2017

\section{Resumen}

Varios estudios de género se ocuparon de las representaciones simbólicas de la mujer en los medios, es decir, la mujer como objeto de los contenidos mediáticos, pero son menos los que analizan su rol como productora de contenidos informativos. Dos investigaciones globales sobre periodismo ofrecen datos para describir las condiciones profesionales de las periodistas en Argentina. La contextualización con estudios similares realizados en otros países permite elaborar un perfil actualizado de las mujeres en la prensa argentina y revisar cuestiones teóricas y metodológicas de los estudios de periodismo que puedan aportar precisiones conceptuales a la discusión que vienen dando los estudios de género sobre este tema.

Palabras Clave: Medios, mujeres, periodistas, estudios de periodismo, diarios, estudios de género.

\begin{abstract}
Gender media researches have focused on women's symbolic representation in the media, that is to say, women as object of media contents. In some cases, the research looks for data about participation of women as news producer. Data from global journalism studies describe professional conditions of women journalists in Argentina. These results will allow comparisons among several countries in order to elaborate an accurate profile of women in argentine press. Besides, this article would like to review some theoretical and methodological concepts of journalism studies to enrich gender studies perspective.
\end{abstract}

Keywords: Media, women, journalists, journalism studies, newspapers, gender studies. 


\section{Las periodistas en el periodismo}

La presencia de la mujer en los medios de comunicación ha sido señalada como un indicador de su jerarquía subordinada en la división del trabajo (Bourdieu, 2000), pero esta observación ha sido estudiada principalmente desde el análisis de su presencia en los programas o las noticias. En cambio, son escasos los estudios que profundizan el estudio de la mujer como productora de contenidos mediáticos, es decir, como "sujeto de semiosis" (Femenías, 2000: 67). El Proyecto de Monitoreo Global de Medios (GMMP) viene señalando la inequidad laboral que experimentan las periodistas y los roles estereotipados de la mujer en los medios informativos (World Association for Christian Communication 2015). Desde 1995 y cada cinco años produce un informe que abarca más de cien países que sin ser una investigación académica, ha sido base de la mayoría de los análisis del tema (Altés, 2006; North, 2016; Ross et al. 2016; Ross y Carter 2016). En el caso particular de Argentina, las investigaciones en esta línea se concentraron en el tratamiento que los medios hacen de las mujeres y sus potenciales efectos en las audiencias mayormente desde el marco conceptual de la semiótica y los estudios del discurso (Laudano, 2010; Rovetto, 2012). Esta orientación, directa o indirectamente, puso el foco en los mensajes más que en quienes los producen, por lo que sigue siendo una incógnita la participación de la mujer en el mercado de trabajo de los medios argentinos

Aunque los estudios de periodismo son el campo más prolífico dentro de la comunicación, especialmente en los últimos cincuenta años (Humanes, 2003) han tenido escaso y tardío desarrollo en Argentina (Arrueta, 2010). Recién en este siglo aparecen trabajos que se ocupan de las prácticas en las redacciones argentinas con marcos conceptuales y métodos propios de los estudios del periodismo (Amado y Waisbord, 2015; Boczkowski, 2010; Dessein y Roitberg, 2014; Luchessi y Videla, 2016; Mancini, 2013; Martini y Luchessi, 2004; Mitchelstein y Boczkowski, 2009; Rost y Liuzzi, 2012). Hasta entonces el periodismo se abordaba desde análisis discursivo de noticias o desde la economía de los medios, enfoques que no consideran las particularidades de la sociología de la profesión (Waisbord, 2013) ni son suficientes para describir la situación profesional de la mujer en el periodismo.

En un contexto donde prácticamente no hubo estudios para conocer la estructura y dinámica de la profesión periodística en general, el problema de la participación de las mujeres quedó restringido a la discusión de los colectivos de periodismo con perspectiva de género (Chaer y Santoro, 2007; Santoro y Chaer, 2010). Estas iniciativas promovieron la producción de contenidos más igualitarios desde la difusión de la problemática de la mujer en los medios y de pautas para una cobertura informativa con perspectiva de género. De ahí que, en contraste con la producción académica sobre rutinas de las periodistas de otros países, en Argentina se produjeron "investigaciones basadas en la perspectiva de los estudios culturales y semióticos que articulan sus análisis con la teoría de género, diversidad sexual, el feminismo y las propias prácticas profesionales. En esta línea de acción se vienen incrementando los monitoreos locales y las 
propuestas para crear conciencia sobre el derecho de las mujeres y la equidad en los medios de comunicación" (Rovetto, 2012: 23).

En línea con esas iniciativas, se promovieron varios instrumentos legales y jurídicos para proteger los derechos humanos de las mujeres con relación a su representación en los medios de comunicación, con foco en la sanción de la "violencia simbólica". En 2009 se sancionaron la Ley 26.485 de Protección Integral para Prevenir, Sancionar y Erradicar la Violencia contra las Mujeres en los Ámbitos en que Desarrollen sus Relaciones Interpersonales y la Ley 26.522 de Servicios de Comunicación Audiovisual, que incluía artículos para exigir el tratamiento plural igualitario y no estereotipado y evitar toda discriminación por género u orientación sexual. Anterior a la ley es el Observatorio de discriminación de radio y televisión, creado en 2006, conformado por las sucesivas autoridades de aplicación de la ley del sector audiovisual, el Instituto Nacional contra la Discriminación, la Xenofobia y el Racismo y el Consejo Nacional de las Mujeres. En su sitio web ${ }^{1}$ solo se detectaron cuatro informes orientados a la labor periodística limitados a la descripción de situaciones concretas que merecieron las recomendaciones del organismo. Estos instrumentos legales se concentraron en la inclusión en los contenidos de la mujer (la mujer en las noticias, en la ficción, en las publicidades) sin acción equivalente que la considerara en tanto productora de esos contenidos. Es decir, que el estudio de la mujer periodista desde sus problemas profesionales y laborales sigue pendiente.

La falta de datos sobre la situación laboral de los periodistas de todo el país y la inexistencia de censos profesionales no permite conocer cuáles son las condiciones laborales del conjunto, lo que explica que no se conozcan las diferencias en la carrera profesional de hombres y mujeres periodistas, más allá de algunos testimonios personales o estudios de caso. A la falta de estadísticas sectoriales y de estudios académicos que permitieran elaborar un perfil profesional general, hay que sumar la debilidad de las instituciones profesionales a la hora de explicar estas carencias (Amado y Waisbord, 2015). Que la mayor cantidad de estudios sobre las periodistas en lengua hispana sean españoles (Rovetto, 2012) se explica en gran medida porque "Las asociaciones de la prensa, los colegios profesionales, los sindicatos o diversos institutos y centros públicos (como el Centro de Investigaciones Sociológicas o el Instituto de la Mujer) han ejercido de patrocinadores para la realización y difusión posterior de investigaciones sobre el perfil de los periodistas y sus prácticas en España” (Soriano, Cantón y Díez, 2005: 37). En Argentina la pertenencia a asociaciones profesionales y sindicatos es baja en general, como lo es en toda América Latina donde solo uno de cada tres periodistas pertenece a alguna asociación profesional (Amado et al., 2016:14). Esto explica la falta de contención profesional que padece el periodismo argentino en general y la poca atención a la situación de las periodistas en particular.

Los estudios internacionales reconocen diferencias entre hombres y mujeres en cuanto a actitudes y criterios profesionales, que estas se explican más por cuestiones estructurales que por el plano individual en el que se inscriben la identidad sexual y las creencias políticas (Ross, 2001; Weaver y Willnat, 2012). Estos estudios coinciden con los teóricos del periodismo que señalan

\footnotetext{
${ }^{1}$ Ente Nacional de Comunicaciones (n.d.).
} 
menor incidencia de las influencias del nivel individual (Shoemaker y Reese 1996) dentro de las que se ubicaría la variable género entendida como identidad subjetiva (Scott, 1996). En cualquier caso, para obtener conclusiones de la relación entre la participación de la mujer en las redacciones y las potenciales influencias (Craft y Wanta, 2004), es necesario identificar previamente los factores del contexto en el que se desempeña. Por ejemplo, la presunción de que la concentración económica de los conglomerados mediáticos son un factor adverso al desarrollo del periodismo con perspectiva de género (Rovetto, 2013), necesita contrastarse con datos concretos que permita comparar la equidad de género por tipo de propiedad de medios, para lo que es necesario conocer la distribución en los medios del país y datos demográficos de los y las periodistas, no elaborados a la fecha (Amado y Pizzolo, 2014).

Este artículo tiene como objetivo principal aportar datos empíricos para identificar el lugar profesional de la mujer en los medios informativos de la Argentina, a los fines de elaborar un perfil de las periodistas argentinas que sirva para futuros análisis desde una perspectiva de género basado en evidencias de campo. Se trata de una descripción preliminar del lugar de la mujer en el periodismo argentino a partir de datos provenientes de dos investigaciones diseñadas para el estudio de la cultura periodística global. Si bien se trata de datos producidos desde una metodología y un marco conceptual de los estudios del periodismo, ofrecen evidencias valiosas para entender la inserción de la mujer en este tipo de organizaciones económicas, lo que se encuadra dentro de uno de los factores para analizar las relaciones de género (Scott, 1996). Se entiende que "Para explicar el hecho de que las mujeres, en la mayoría de las sociedades conocidas, están consignadas a posiciones sociales inferiores, es necesario tomar en cuenta la asimetría de posiciones adscritas a cada género en la economía de los intercambios simbólicos" (Lamas, 1999: 162). Por lo tanto, se propone como un aporte inicial de datos y precisiones técnicas para estudiar las mujeres que ejercen la profesión periodística, que puedan ser luego discutidos y enriquecidos por las especialistas en el tema género.

El discurso de la femineidad en los medios "no sólo está inmerso en las relaciones económicas y sociales, sino que también constituye 'un conjunto de relaciones' que surgen en "marcos históricos locales"” (West, Lazar, y Chramare, 2000: 184). En este sentido, el periodismo como profesión es un objeto de estudio situado en contextos políticos, económicos y sociales, en tanto que "Professions do not exist in isolation; they are permanently engaged in relations with other social fields. This perspective differs from conventional, taxonomic analysis that views professionalism in terms of certain attributes that, in principle, are found in archetypical professions" (Waisbord, 2013: 11). Por ello, se analizará a las mujeres periodistas como parte de una profesión en cambio, atravesada por tensiones sociales y políticas que hace del periodismo una etiqueta vulgarmente aplicada a una diversidad de ocupaciones. Las particularidades propias de un campo profesional pueden enriquecer la perspectiva de género y evitar confusiones conceptuales como las que tratan indistintamente medios y periodistas como si trataran de actores similares (Hassan y Gil, 2014), siendo que tienen problemáticas muy distintas y metodologías de estudio muy diferentes. De ahí que el objetivo secundario de este artículo sea aportar definiciones conceptuales del campo del periodismo que puedan ser útiles para los estudios de la profesión 
con perspectiva de género y permitan contar con una descripción del conjunto para comprender mejor la inserción de la mujer en la actividad periodística.

Los artículos sobre el periodismo con perspectiva de género en Argentina (Chaer, 2007; Laudano, 2010; Rovetto, 2012, 2013) proponen algunas conclusiones que se tomarán como hipótesis de partida para analizar la participación de las periodistas argentinas en la producción de las noticias:

H1: Las periodistas argentinas tienen una participación minoritaria en los medios argentinos, donde están relegadas a posiciones subalternas.

H2: Las periodistas argentinas se ocupan de temas accesorios a la agenda noticiosa de las secciones centrales de los periódicos, mientras los hombres dominan las secciones duras.

Estas dos afirmaciones van a ser contrastadas con el perfil profesional de las mujeres en los medios argentinos y las pautas que se les asigna en la prensa elaborados a partir de las bases de datos de dos estudios globales de periodismo que aportan evidencias empíricas del colectivo, de los cuales se toman los datos que permitan analizar el lugar de la mujer en los medios argentinos.

\section{Metodología y hallazgos: Estudiar las periodistas desde el periodismo}

El presente artículo propone analizar los datos obtenidos a partir de dos estudios globales de periodismo que contaron con un capítulo argentino. ${ }^{2}$ El primero es el estudio Worlds of Journalism (WJS, 2016) destinado a estudiar la cultura periodística y los factores de influencia a nivel global. El estudio global relevó las condiciones profesionales de 27500 periodistas en 66 países, dentro de los que se incluyeron siete de Latinoamérica (Argentina, Brasil, Chile, Colombia, Ecuador, El Salvador y México). ${ }^{3}$ El estudio definió como periodistas a las personas que tuvieran como ingreso principal el trabajo de producción y la edición de contenidos o

\footnotetext{
${ }^{2}$ Las investigaciones formaron parte de los proyectos de investigación de la Universidad Nacional de La Matanza, de Buenos Aires, dentro del marco del programa PROINCE entre 2012 y 2015. El artículo fue elaborado como parte de la estancia académica en la Universidad de Castilla La Mancha en 2017.

${ }^{3}$ La muestra latinoamericana incluyó 438 medios de comunicación, relevados durante los años 2013 y 2015, que representa una muestra de 2789 periodistas para un 95\% de confianza y un margen de error del 5\% (Amado et al., 2016). En ese marco se aplicó una encuesta nacional por medio de entrevistas personales a 363 periodistas de Argentina de acuerdo a un cuestionario cerrado, entre octubre de 2013 y diciembre de 2014 (Amado, 2016). Dado que no existen registros oficiales de profesionales o de medios en la Argentina, la muestra local tomó como unidad muestral agregada los medios de comunicación de Argentina que poseen sus propios programas o secciones de noticias a partir de un listado nacional elaborado por un observatorio de medios privado. Se realizó un muestreo polietápico a partir de una selección por conglomerados y estratificada (tipo, propiedad, alcance y localización física del medio, y datos censales de la población general). Los profesionales entrevistados se seleccionaron por cadena de bola de nieve para subsanar las dificultades para la selección y localización.
} 
supervisión y coordinación editorial de medios de comunicación. Dentro de las variables demográficas se incluía la identificación hombre o mujer, según era proporcionada por el o la profesional entrevistados que permite la construcción de los indicadores que aquí se presentan.

El segundo insumo es el estudio global Journalistic Role Performance, ${ }^{4}$ basado en un análisis de contenido para una muestra de noticias del cuerpo principal de diarios. Este estudio compara la puesta en práctica de distintos roles periodísticos a partir de un análisis de contenido de noticias en la prensa nacional de países con diferentes contextos políticos y sociales (Mellado, 2015) . El $^{5}$ estudio busca identificar variables para operacionalizar la "performance" periodística en el contenido a través de tres aspectos que aparecen en las noticias: la presencia de la voz del periodista en las noticias; las relaciones de poder; y la forma en que el periodismo aborda a la audiencia. La codificación incluyó otros elementos como fuentes, temas, formatos, que se tomarán para el análisis de la presencia de mujeres como autoras de las noticias. La selección de diarios se basó en criterios de orientación ideológica y de mercado, de modo de contar con diversidad editorial que permitiera la comparación con los diarios de los otros países participantes en el estudio.

\section{Tabla 1. Distribución de la muestra de diarios argentinos analizados en el estudio Journalistic Role Performance}

\begin{tabular}{|l|c|c|c|c|}
\hline Variable / Medio & Clarín & Diario Popular & Página 12 & La Nación \\
\hline Orientación ideológica & Centro & Centro & $\begin{array}{c}\text { Centro } \\
\text { Izquierda }\end{array}$ & $\begin{array}{c}\text { Centro } \\
\text { Derecha }\end{array}$ \\
\hline Orientación de mercado & Popular & Popular & Elite & Elite \\
\hline $\begin{array}{l}\text { Ejemplares vendidos, promedio } \\
2012 / 2013 \quad \begin{array}{c}\text { (según Instituto } \\
\text { Verificador de Circulaciones) }\end{array}\end{array}$ & 270000 & 88000 & S/D & 165000 \\
\hline Cantidad de noticias analizadas & 1247 & 669 & 802 & 682 \\
\hline
\end{tabular}

Fuente: Elaboración propia con datos inéditos del estudio Journalistic Role Performance, capítulo Argentina $^{6}$

\footnotetext{
${ }^{4}$ Los aspectos metodológicos y el alcance del estudio pueden consultarse en Journalistic Role Performance around the Globe (n.d.). Los datos surgen de procesar la base de datos correspondiente a Argentina, no publicada.

${ }^{5}$ La muestra para el estudio de Argentina se tomó en cuatro diarios de referencia desde el 2 de enero de 2012 al 31 de diciembre de 2013, mediante el método de semana construida que permite establecer una muestra representativa para los dos años analizados. Con los días seleccionados aleatoriamente se armaron dos semanas construidas por año, una para cada uno de los semestres. Se consideró como unidad de análisis cada una de las noticias publicadas el día seleccionado en las secciones de actualidad de los diarios Clarín, La Nación, Página 12 y Diario Popular, excluyendo notas de opinión, editoriales y entrevistas que sean en mayoritariamente preguntas y respuestas. El relevamiento de todas las noticias publicadas en la edición del día seleccionado dio un total de 3400 noticias. Las noticias fueron codificadas manualmente por un equipo supervisado por la coordinación global del estudio que unificó los criterios e instrumentos de análisis de contenido.

${ }^{6}$ Journalistic Role Performance around the Globe (n.d.).
} 


\subsection{Quiénes son las periodistas argentinas}

La falta de datos censales del periodismo argentino no permite conocer las cuotas demográficas de la profesión para contestar la hipótesis primera de manera fehaciente. No obstante, las encuestas de alcance nacional realizadas por la única asociación profesional del país coinciden en sucesivos años en estimar en un tercio la presencia de mujeres (Foro de Periodismo Argentino, 2005, 2011, 2014), sin que se cuenten con datos anteriores. Sin otros antecedentes locales, indagaciones exploratorias concluyen que "a partir de los estudios consultados y las entrevistas realizadas podemos sostener que en las redacciones domina un ambiente muy masculino que se traduce en una cierta cultura periodística que no contempla entre sus presupuestos la dimensión de género" (Rovetto, 2013: 60).

La muestra para Argentina del estudio Worlds of Journalism (WJS) determinó un 36.9\% de mujeres, que ubica a la Argentina en la $49^{\mathrm{a}}$ posición entre los 66 países encuestados en la investigación. Según el informe general de $\mathrm{WJS}^{7}$, diecinueve países cuentan con mayoría de mujeres en las muestras nacionales: Letonia, Rusia, Rumania, Sudáfrica, Moldovia, Singapur, Estonia, Australia, Finlandia, Croacia, Serbia, Malasia, Albania, Filipinas, China, Nueva Zelandia, Noruega, Emiratos Árabes y Bután. Brasil es el país de Latinoamérica con mayor participación de mujeres en la profesión (49.2\%), seguido de Chile (43,2\%); El Salvador (40,4\%); Colombia (39,7\%); Argentina (36,9\%); Ecuador (33,2\%); y México (31,8\%). Después de México, siguen nueve países con menos de un tercio de participación femenina: Sierra Leona, Etiopia, Qatar, India, Estados Unidos, Corea del Sur, Indonesia, Japón y Bangladesh.

Un estudio realizado a fin del siglo pasado entre veinte mil periodistas señalaba el predominio de varones en las redacciones, con marcadas diferencias alrededor del promedio global de $33 \%$ (Weaver 1999: 122). Mientras en algunos países como Nueva Zelanda y Finlandia las mujeres casi igualaban a los hombres, en otros como Corea, Argelia, España y Gran Bretaña la presencia de mujeres quedaba por debajo del 25\%. Años después la participación de las periodistas pasó del tercio del estudio de 1998 a $41 \%$ y si bien los hombres seguían siendo mayoría en 23 de los 29 países incluidos en la investigación, la proporción se equilibraba en Australia, Brasil, Gran Bretaña, Dinamarca, Hong Kong, Hungría, España y Suecia (Weaver y Willnat, 2012: 38). Un dato que destacan los investigadores es que entre 1992 y 2002 no se modificó el porcentaje de 33\% de mujeres periodistas en Estados Unidos, a pesar de la cantidad de mujeres que participan en los programas de periodismo. Esto planteó preguntas acerca de la incidencia de la educación que toman estudios españoles que concluyen que un mayor número de mujeres estudiantes de periodismo no ha redundado en un mayor número de mujeres periodistas en los medios (Rivero Santamarina, 2014). En Latinoamérica también las estudiantes son mayoría en las carreras de comunicación (Gobbi y Betti, 2013) pero esa preponderancia en las casas de estudio no parece reflejarse en los medios. En el caso de Argentina, las cifras del Ministerio de Educación informan de que en 2014 el $57,4 \%$ de estudiantes en el sistema universitario eran mujeres, y el $61,5 \%$ de las egresadas, cifras

\footnotetext{
${ }^{7}$ Worlds of Journalism (2016).
} 
similares a las de 2011 (57,1\% y 61,2\% respectivamente $)^{8}$. En el caso de estudiantes de posgrado, el 58,2\% eran mujeres, que eran el 57,2\% de quienes obtienen la titulación. Las cifras de la encuesta WJS confirman un alto nivel de formación en el periodismo argentino. Tres cuartas partes de los periodistas hombres cuentan con titulación universitaria específica en periodismo (51.4\%) o en otra carrera (22\%), y un $14 \%$ tienen formación de posgrado. En el caso de las mujeres, la titulación universitaria es superior (60\% tienen licenciatura o equivalente en periodismo, y $23 \%$ en otra especialidad), pero las periodistas de la muestra con posgrado (10.9\%) son menos, en proporción, que la de sus colegas masculinos (13,7\%). Quizás la mayor diferencia, aunque mínima en números, es que son mayoría los periodistas varones con formación secundaria o menor (12,3\%) mientras que las mujeres son la mitad $(5,4 \%)$.

El informe GMMP señala para Argentina que la presencia de periodistas en los medios en el día que se hizo el monitoreo era de $15 \%$ de mujeres para prensa, $35 \%$ para radio y $35 \%$ para televisión (World Association for Christian Communication, 2015: 6). Esta observación no guarda correlación con la distribución en medios que tiene la encuesta nacional base de este artículo. Según esta muestra, los medios con mayor equidad son las revistas, las agencias de noticias y la radio (Tabla 2.), medio que fue pionero en el país en el reconocimiento de periodistas y conductoras de programas (Ehrick, 2016) pero poco tratado en los estudios de periodismo y género. En contraste, la televisión y los medios digitales tienen una proporción de tres hombres por cada mujer periodista. Estos resultados plantean la dificultad de unificar los medios como un conjunto homogéneo a la hora de hablar de la participación de la mujer.

Tabla 2. Distribución de periodistas mujeres y hombres de la muestra de periodistas entrevistados en Argentina para la encuesta global Worlds of Journalism (WJS) (N=353 respuestas)

\begin{tabular}{|l|c|c|c|c|c|}
\hline & Mujeres & \% del medio & Hombres & \% del medio & $\begin{array}{c}\text { Total periodistas por } \\
\text { medio }\end{array}$ \\
\hline Diarios & 34 & $39,1 \%$ & 53 & $60,9 \%$ & 87 \\
\hline Semanarios & 1 & $5,6 \%$ & 17 & $94,4 \%$ & 18 \\
\hline Revistas & 8 & $53,3 \%$ & 7 & $46,7 \%$ & 15 \\
\hline Televisión & 11 & $23,4 \%$ & 36 & $76,6 \%$ & 47 \\
\hline Radio & 40 & $44,4 \%$ & 50 & $55,6 \%$ & 90 \\
\hline Agencia de noticias & 23 & $46,0 \%$ & 27 & $54,0 \%$ & 50 \\
\hline $\begin{array}{l}\text { Medio digital } \\
\text { independiente }\end{array}$ & 10 & $25,6 \%$ & 29 & $74,4 \%$ & 39 \\
\hline $\begin{array}{l}\text { Medio digital de } \\
\text { medio tradicional }\end{array}$ & 2 & $28,6 \%$ & 5 & $71,4 \%$ & 7 \\
\hline & 129 & $36,5 \%$ & 224 & $63,5 \%$ & 353 \\
\hline
\end{tabular}

Fuente: Elaboración propia con datos de Worlds of Journalism Study, capítulo Argentina (Amado, 2016)

\footnotetext{
${ }^{8}$ Ministerio de Educación de la Argentina (2011, 2014).
} 
Los datos también permiten cuestionar ciertas presunciones con relación al esquema de propiedad en tanto que como los colectivos que promueven el periodismo de género se desarrollan en medios comunitarios, se da por hecho de que son espacios más propicios para el ejercicio de la profesión. Sin embargo, los medios comunitarios no parecen ser todavía un espacio de desarrollo profesional más propicio para las mujeres si se entiende como profesión aquella que brinda el sustento principal, como requería el estudio. El relevamiento nacional de más de mil doscientos periodistas para seleccionar la muestra que encajara en los parámetros del estudio, puso en evidencia que la mayoría de los periodistas que trabajan en medios comunitarios lo hacen de manera eventual, teniendo como trabajo principal otro, que en muchos casos no tiene que ver con el periodismo. Este punto no es menor en la discusión sobre la conquista de espacios laborales de la mujer, ancestralmente relegada a tareas no remuneradas o a desarrollar su vocación de manera tangencial a aquello que le da sustento.

El promedio latinoamericano muestra una diferencia en este punto. En los datos generales el 60,3\% del total de la muestra latinoamericana son hombres, porcentaje similar al de Argentina. Sin embargo, la diferencia es que la proporción se mantiene en los medios privados y estatales, con un leve aumento en el caso de los medios de la sociedad civil tipificados como públicos en WJS, en los que la proporción de mujeres llega al 45,9 \% (Amado et al., 2016: 12).

\section{Gráfico 1. Porcentaje de periodistas mujeres y hombres según tipo de medio (privado, público, estatal) de la muestra del estudio WJS ( $=359)$}

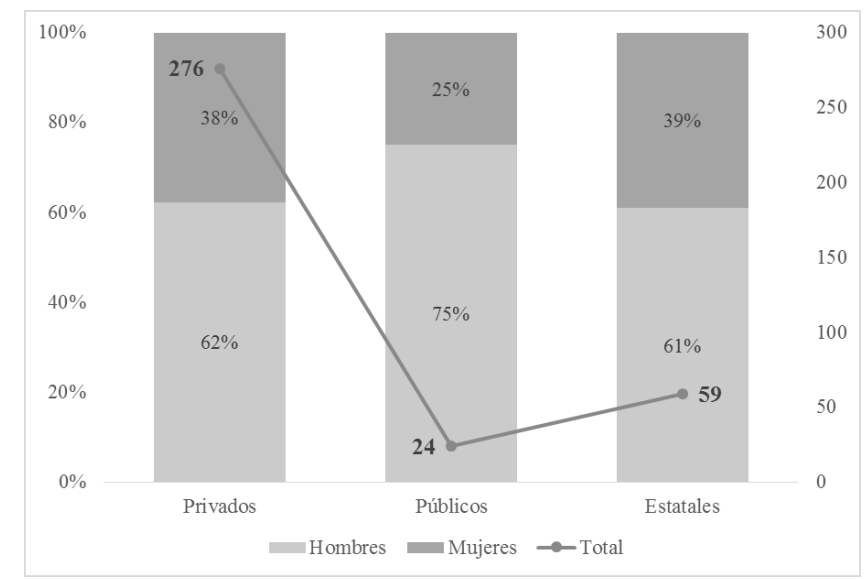

Fuente: Elaboración propia con datos de Worlds of Journalism Study, capítulo Argentina (Amado, 2016)

La subrepresentación de las mujeres es mayor en los puestos directivos. Un estudio señalaba que en los cinco diarios de mayor tirada en España las mujeres eran el 14\% de los puestos de decisión (citado por Rovetto, 2012:19). Investigaciones de las televisoras autonómicas de ese país muestran que la participación en cargos jerárquicos puede oscilar entre el $42 \%$ y el $12 \%$ (Soriano et al., 2005:41). Hacia 1995 solo el 3\% de las organizaciones de medios mundiales eran dirigidas 
por mujeres, cifra que hacia 2011 ascendió a 27\% entre 500 compañías de medios en 60 países, y que es del $41 \%$ en los cargos de editoras (North, 2016).

La cifra es similar al porcentaje de mujeres en los medios lo que parecería indicar que el problema en estos años no fue el ascenso en el escalafón sino la participación en general en esa industria. Los datos de Argentina señalan mayoría de mujeres en ciertas posiciones, como la de productora y redactora y aunque el porcentaje en la dirección general de los medios es más alto en los hombres, la distribución se equilibra en los cargos medios de editores. Para poder hacer una comparación de la distribución de cargos se hizo un cálculo del porcentual que correspondían los cargos de las 132 mujeres y los 228 hombres que identificaron su cargo.

Tabla 3. y Gráfico 2. Distribución porcentual sobre el total de cargos de la muestra del estudio WJS en Argentina ( $\mathrm{N}=360)$

\begin{tabular}{|l|l|l|}
\hline & Varones & Mujeres \\
\hline Director/a & $18,0 \%$ & $5,3 \%$ \\
\hline Editor/a general & $7,5 \%$ & $6,1 \%$ \\
\hline Editor/a Jefe/a Sección & $10,1 \%$ & $7,6 \%$ \\
\hline Editor/a Jefe/a Subsección & $0,9 \%$ & $1,5 \%$ \\
\hline Editor/a & $9,2 \%$ & $11,4 \%$ \\
\hline Productor/a & $12,3 \%$ & $18,9 \%$ \\
\hline Reportero/a & $15,4 \%$ & $12,9 \%$ \\
\hline Redactor/a & $19,7 \%$ & $26,5 \%$ \\
\hline Pasante & $0,4 \%$ & $3,8 \%$ \\
\hline Otros cargos & $6,6 \%$ & $6,1 \%$ \\
\hline & $100 \%$ & $100 \%$ \\
\hline
\end{tabular}

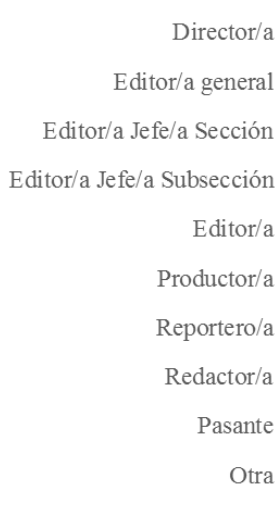

Editor/a general

Subsección

Editor/a

ductor/a

Reportero/a

dactor/a

Otra

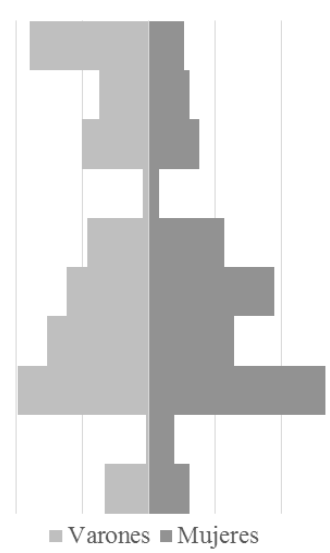

Fuente: Elaboración propia con datos de Worlds of Journalism Study, capítulo Argentina (Amado, 2016)

Asociado a la escala jerárquica, los ingresos son otro factor importante a la hora de mensurar la brecha profesional entre hombres y mujeres (Soriano et al., 2005). El cuestionario global indagaba sobre el ingreso, que después se convirtió a dólares norteamericanos y clasificó en una escala de diez categorías. La pirámide que acompaña Tabla 4. muestra una distribución hacia los extremos. Más de la mitad de la muestra gana menos de U\$D 2.500, franja salarial que comprende el $60.8 \%$ de las mujeres y el 60,2\% de los hombres, lo que equipara los dos grupos en un problema que se manifiesta como estructural.

La diferencia salarial se observa en el tercio más bajo y en el más alto, donde se invierten levemente las proporciones. Dentro del tercio que gana menos de U\$D 1.500 se ubican un $32.8 \%$ de las mujeres y un 25\% de los varones. En el tercio superior están los que ganan más de U\$D 6.000, y ahí la proporción se invierte en tanto que se ubican el 23,4\% de las mujeres y el 31,8\% de los hombres. Esta franja es muy numerosa y contiene funciones de distintas jerarquías. Las categorías que más declaran ese ingreso son la de productor y redactor, y cada una de estas 
categorías duplica en cantidad de profesionales a los directores con ese nivel salarial. El nivel de ingreso superior fue declarado por un tercio de los entrevistados que se desempeñan en los medios privados (30\%), otro tanto de los medios estatales (28\%), y un $20 \%$ de los medios comunitarios. Esta variación salarial confirma la baja influencia sindical en el periodismo argentino, que carece de escalas salariales reguladas por convenios. Pero también habla de la existencia de una elite favorecida por los negocios y por pauta publicitaria en los tres tipos de medios, como develaba las asignaciones abultadas de muchos periodistas que aparecían en el listado de beneficiarios de publicidad del Poder Ejecutivo Nacional (Waisbord y Amado, 2014).

Tabla 4. y Gráfico 3. Distribución del nivel de ingresos en porcentaje del total de los periodistas por género de la muestra del estudio WJS $(\mathbf{N}=\mathbf{2 8 0})$

\begin{tabular}{|l|l|l|}
\hline Salario U\$D & Mujeres & Varones \\
\hline Más de 6000 & $23,40 \%$ & $31,80 \%$ \\
\hline $5001-6000$ & $2,80 \%$ & $1,10 \%$ \\
\hline $4001-5000$ & $6,50 \%$ & $5,10 \%$ \\
\hline $3001-4000$ & $6,50 \%$ & $1,70 \%$ \\
\hline $2501-3000$ & $0,90 \%$ & $1,70 \%$ \\
\hline $2001-2500$ & $15,90 \%$ & $17,00 \%$ \\
\hline $1501-2000$ & $11,20 \%$ & $16,50 \%$ \\
\hline $1001-1500$ & $17,80 \%$ & $10,80 \%$ \\
\hline $501-1000$ & $10,30 \%$ & $10,80 \%$ \\
\hline $0-500$ & $4,70 \%$ & $3,40 \%$ \\
\hline & $100,00 \%$ & $100,00 \%$ \\
\hline
\end{tabular}

$$
\begin{array}{r}
\text { Más de } 6000 \\
5001-6000 \\
4001-5000 \\
3001-4000 \\
2501-3000 \\
2001-2500 \\
1501-2000 \\
1001-1500 \\
501-1000 \\
0-500
\end{array}
$$

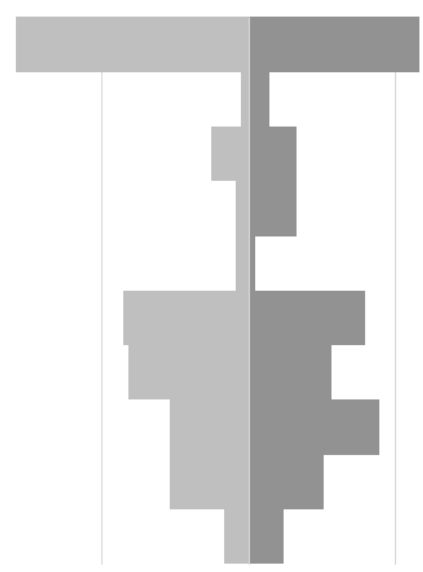

$\llbracket$ Varones $\square$ Mujeres

Fuente: Elaboración propia con datos de Worlds of Journalism Study, capítulo Argentina (Amado, 2016)

\subsection{De qué se ocupan las periodistas argentinas}

La segunda hipótesis a constatar en este análisis se basa en observaciones previas acerca del lugar subsidiario que ocupa la mujer en los medios (North, 2016; Ross et al., 2016). En México recién en este siglo se consolidó la posición de las mujeres en las secciones de información general, aunque sigue siendo relegada en puestos directivos y en la sección de opinión (Valles Ruiz, 2006). El estudio GMMP señala desigualdad entre quienes reportan las noticias: "salvo en los casos de ciencia y salud y celebridades, deportes y artes, la cantidad de noticias reportadas por hombres es mayor. Dentro de las noticias que abordan las mujeres, crimen y violencia muestra el número más alto y economía el más bajo, sólo 10\%" (World Association for Christian Communication, 2015:13). La comparación con las especialidades declaradas por los periodistas de la muestra WJS 
muestra coincidencia parcial con esos resultados. Del total de la muestra entrevistada, un tercio trabajaba en secciones específicas $(34,2 \%)$ mientras que la mayoría no se dedicaba a una única especialidad. Entre las especialidades no se observan mayores diferencias entre mujeres y varones, con excepción de las secciones de Policiales y Deportes, con predominio de hombres.

\section{Gráfico 4. Secciones área o sector informativo en que trabajan normalmente periodistas mujeres y hombres especializados en una sección $(\mathrm{N}=124)$}

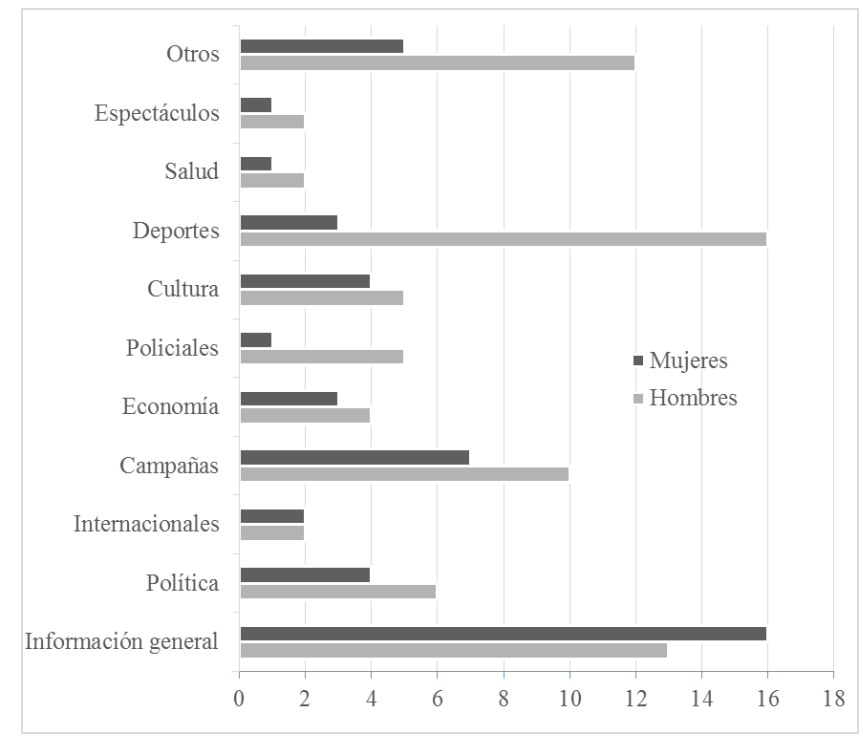

Fuente: Elaboración propia con datos de Worlds of Journalism Study, capítulo Argentina (Amado, 2016)

El monitoreo de la World Association of Christian Communication se concentra en un solo día del año, con lo que la cobertura noticiosa puede estar sujeta a desvíos propios de la agenda del día. En el estudio de Journalistic Role Performance se relevaron 3400 noticias de cuatro diarios en una muestra representativa de todos los meses y todos los días de los años 2012 y 2013. Con esta base, sus conclusiones son válidas para los diarios seleccionados, que son referenciales en la prensa gráfica nacional. De las noticias analizadas, un tercio (29\%) corresponden a notas firmadas $(\mathrm{N}=986)$, que ofrecen un corpus para indagar la proporción de hombres y mujeres. Este porcentaje es similar al de la participación femenina en los medios en general pero el 15\% de notas firmadas por mujeres que da el informe GMMP para Argentina es la mitad que la detectada en los diarios analizados, que es del 32,7\% (Gráfico 5).

La proporción disminuye si se toma el conjunto de las notas de los diarios, en las que las firmas de nombres de mujeres representan apenas una de cada diez de las notas del periódico $(9,5 \%)$, la mitad que las que firman los hombres. El resto corresponde a notas sin firma o firmadas por agencias o el equipo de la redacción, en donde no se puede determinar el género de la autoría. 
El Diario Popular tiene muy baja proporción de firmas entre sus notas y de las 13 identificadas en la muestra de 3400 noticias no hubo ninguna firma de mujeres. Le sigue en baja proporción el diario Página/12, donde las firmas de mujeres representan un cuarto del total de notas firmadas.

Gráfico 5. Notas firmadas en cuatro diarios durante 2012-2013 (N=3400)

\begin{tabular}{|l|l|l|l|l|l|l|l|}
\hline & $\begin{array}{l}\text { Notas } \\
\text { firmadas por } \\
\text { mujeres }\end{array}$ & $\begin{array}{l}\text { \% de } \\
\text { firmas } \\
\text { femeninas }\end{array}$ & $\begin{array}{l}\text { notas } \\
\text { firmadas } \\
\text { por } \\
\text { hombres }\end{array}$ & $\begin{array}{l}\text { \% de } \\
\text { firmas } \\
\text { masculinas }\end{array}$ & $\begin{array}{l}\text { Total } \\
\text { notas } \\
\text { firmadas }\end{array}$ & $\begin{array}{l}\text { \% firmas } \\
\text { femeninas } \\
\text { sobre el total } \\
\text { de la muestra }\end{array}$ & $\begin{array}{l}\text { Total } \\
\text { muestra }\end{array}$ \\
\hline La Nación & 134 & $36,1 \%$ & 237 & $63,9 \%$ & 371 & $16,7 \%$ & 802 \\
\hline Clarín & 143 & $33,6 \%$ & 283 & $66,4 \%$ & 426 & $11,5 \%$ & 1247 \\
\hline Página/12 & 45 & $25,6 \%$ & 131 & $74,4 \%$ & 176 & $6,6 \%$ & 682 \\
\hline $\begin{array}{l}\text { Diario } \\
\text { Popular }\end{array}$ & 0 & $0 \%$ & 13 & $100 \%$ & 13 & $0,0 \%$ & 669 \\
\hline Total & 322 & $32,7 \%$ & 664 & $67,3 \%$ & 986 & $9,5 \%$ & 3400 \\
\hline
\end{tabular}

Fuente: Elaboración propia con datos inéditos del estudio Journalistic Role Performance, capítulo Argentina $^{9}$

El resultado es llamativo porque el diario Página 12 es considerado un medio identificado con la perspectiva de género y la agenda de la mujer. Por eso el caso es interesante para repensar la relación entre los contenidos y las condiciones laborales de quienes los producen. Aunque $L a$ Nación y Clarín tienen suplementos semanales dedicados a la mujer, el reconocimiento que merece el suplemento que edita Página 12 es muy diferente en cuanto al tratamiento de la perspectiva de género:

“Clarín Mujer” y "Las/12" son dos casos paradigmáticos del tipo de suplemento llamado de la misma manera, "femenino", pero con propuestas diferentes. A grandes rasgos, podría decirse que mientras "Clarín Mujer", editado por el diario Clarín, tiene una propuesta similar a la de las clásicas revistas femeninas -moda, estética, consejos, entrevistas livianas, notas sociales y/o políticas "suaves", solidaridad-, "Las/12" apuesta al público habitual del diario Página 12 con una propuesta más comprometida. Desde un ideario feminista sostenido por el staff que lo realiza, el suplemento nacido hace diez años apuesta al cuestionamiento de la sociedad patriarcal y de los estereotipos sexistas. Ambos comparten, sin embargo, la línea ya mencionada de visibilización de las mujeres y, sólo en algunos casos, "Las/12" incursiona en una perspectiva relacional mujervarón para realizar periodismo de género (Chaher, 2007: 112).

Esta contradicción entre la ideología del medio y las políticas laborales es frecuente dentro del periodismo, y plantea la necesidad de indagar en otros factores del relegamiento de la mujer en el cuerpo principal del diario, más allá de la visibilidad de la problemática de género en las noticias.

\footnotetext{
${ }^{9}$ Journalistic Role Performance around the Globe (n.d.).
} 
De hecho, varían de diario a diario los distintos temas que en el cuerpo principal de los diarios son asignados a las mujeres. Por empezar, hay que recordar que el Diario Popular no figura en la Tabla 5. porque no tenía ninguna nota firmada por una mujer entre las 13 que tenían firma ( $\mathrm{N}=669$ ). El diario que le sigue en menor proporción de firmas de mujeres es Página 12, con una leve mayoría de mujeres en temas de campañas políticas y en derechos humanos, categoría en la que el estudio incluyó temáticas como derechos reproductivos, de identidad de género, familia, entre otros que suelen estar en la agenda de la cobertura con perspectiva de género. Este diario muestra igualdad en número de firmas en la cobertura de protestas, salud y problemas sociales y prácticamente una exclusión de firmas de mujeres en economía, religión, energía y gobierno.

Tabla 5. Tema principal de la noticia de las noticias firmadas por mujeres en el estudio Journalistic Role Performance, capítulo Argentina $(\mathrm{N}=973)^{\mathbf{1 0}}$

\begin{tabular}{|l|l|l|l|l|l|l|}
\hline & \multicolumn{2}{|l|}{ Clarín } & \multicolumn{2}{l|}{ La Nación } & \multicolumn{2}{l|}{ Página 12 } \\
\cline { 2 - 7 } & Mujeres & Varones & Mujeres & Varones & Mujeres & Varones \\
\hline Accidentes, desastres naturales & $\mathbf{5 4 , 5 \%}$ & $\mathbf{4 5 , 5 \%}$ & $\mathbf{5 5 , 6 \%}$ & $\mathbf{4 4 , 4 \%}$ & $12,5 \%$ & $87,5 \%$ \\
\hline Campañas, elecciones & $26,5 \%$ & $73,5 \%$ & $38,2 \%$ & $61,8 \%$ & $\mathbf{5 8 , 3 \%}$ & $\mathbf{4 1 , 7 \%}$ \\
\hline Tribunales & $15,8 \%$ & $84,2 \%$ & $35,0 \%$ & $65,0 \%$ & & \\
\hline Defensa & $0,0 \%$ & $100 \%$ & $28,6 \%$ & $71,4 \%$ & & \\
\hline Protestas, demonstraciones & $61,1 \%$ & $38,9 \%$ & $\mathbf{4 2 , 1 \%}$ & $\mathbf{5 7 , 9 \%}$ & $\mathbf{5 0 , 0 \%}$ & $\mathbf{5 0 , 0 \%}$ \\
\hline Economía & $33,8 \%$ & $66,2 \%$ & $11,5 \%$ & $88,5 \%$ & $4,5 \%$ & $95,5 \%$ \\
\hline Educación & $27,3 \%$ & $72,7 \%$ & $80,0 \%$ & $20,0 \%$ & $16,7 \%$ & $83,3 \%$ \\
\hline Energía, medioambiente & $75,0 \%$ & $25,0 \%$ & $\mathbf{5 0 , 0 \%}$ & $\mathbf{5 0 , 0 \%}$ & $0,0 \%$ & $100,0 \%$ \\
\hline Gobierno & $22,2 \%$ & $77,8 \%$ & $34,0 \%$ & $66,0 \%$ & $9,3 \%$ & $90,7 \%$ \\
\hline Salud & $\mathbf{5 5 , 6 \%}$ & $\mathbf{4 4 , 4 \%}$ & $66,7 \%$ & $33,3 \%$ & $\mathbf{5 0 , 0 \%}$ & $\mathbf{5 0 , 0 \%}$ \\
\hline Vivienda, infraestructura & $\mathbf{4 6 , 7 \%}$ & $\mathbf{5 3 , 3 \%}$ & $28,6 \%$ & $71,4 \%$ & & \\
\hline Derechos humanos & $33,3 \%$ & $66,7 \%$ & $100 \%$ & $0 \%$ & $61,5 \%$ & $38,5 \%$ \\
\hline Otros & $\mathbf{4 4 , 4 \%}$ & $\mathbf{5 5 , 6 \%}$ & $\mathbf{5 6 , 0 \%}$ & $\mathbf{4 4 , 0 \%}$ & $16,7 \%$ & $83,3 \%$ \\
\hline Policiales & $29,1 \%$ & $70,9 \%$ & $26,8 \%$ & $73,2 \%$ & $37,9 \%$ & $62,1 \%$ \\
\hline Religiones & $33,3 \%$ & $66,7 \%$ & $\mathbf{5 0 , 0 \%}$ & $\mathbf{5 0 , 0 \%}$ & $0,0 \%$ & $100,0 \%$ \\
\hline Problemas sociales & $66,7 \%$ & $33,3 \%$ & $40,0 \%$ & $60,0 \%$ & $\mathbf{5 0 , 0 \%}$ & $\mathbf{5 0 , 0 \%}$ \\
\hline Transporte & $28,6 \%$ & $71,4 \%$ & $\mathbf{5 8 , 3 \%}$ & $\mathbf{4 1 , 7 \%}$ & $20,0 \%$ & $80,0 \%$ \\
\hline Total general & $33,6 \%$ & $66,4 \%$ & $36,1 \%$ & $63,9 \%$ & $25,6 \%$ & $74,4 \%$ \\
\hline
\end{tabular}

Fuente: Elaboración propia con datos inéditos del estudio Journalistic Role Performance, capítulo Argentina ${ }^{11}$

${ }^{10}$ Grisado, participación mayoritaria; en negrita, distribución equilibrada de mujeres y hombres.

${ }^{11}$ Journalistic Role Performance around the Globe (n.d.). 
El diario Clarín muestra cierto equilibrio de firmas en las notas que tienen como tema central salud, infraestructura, cobertura de desastres y otros (categoría que incluía cuestiones de estilo de vida, deportes, espectáculos), y mayoría femenina en protestas, energía y medio ambiente y problemas sociales. El diario La Nación, que es el que más proporción de firmas de mujeres ofrece, incorpora a la mujer en rubros que en los otros diarios son mayormente asociados a varones como transporte, educación y religión, además de mantener el equilibrio en temas de protestas, salud, energía y medio ambiente, y accidentes naturales.

La opinión extendida es que las notas con perspectiva de género se suelen ubicar en las secciones conocidas como blandas, "es decir, aquellas en las que se coloca la información considerada menos importante: Sociedad, Espectáculos, Sociales, Policiales. En Política, Internacionales, Economía (llamadas secciones "duras") las notas parecieran no tener género" (en Chaer y Santoro, 2007: 130). Esta presunción se mantiene en algunos estudios que observaron que en la conducción de los programas nocturnos hay más hombres, mientras que en los matinales y de fin de semana imperan las mujeres, de donde se sigue que el periodismo "duro" y de calidad se asocia con los hombres, a los que se ceden las franjas horarias más informativas y menos sensacionalistas (citado por Rivero Santamarina, Meso Ayerdi, y Peña Fernández 2015: 573).

Economía, defensa, gobierno y policiales parecen ser los temas en que se mantiene el predominio de autoría de hombres en los tres diarios analizados, pero no puede decirse que sean los únicos que pueden clasificarse como temas duros. Esta distinción entre noticias duras y blandas se basa mayormente en conceptos analíticos propuestos por los investigadores, más que categorías que usen taxativamente los periodistas, que las asignan de manera más intuitiva que técnica (Tuchman, 1978). El recorrido teórico parece indicar que el concepto duro o blando no necesariamente está dado por la naturaleza de los hechos reportados sino en los factores sociales que impactan en la producción de noticias. Es frecuente encontrar en la literatura la asociación de soft news con noticias frívolas, asociando el significado de liviano que tiene el término en inglés. Sin embargo, el concepto teórico de hard news está vinculado con aquellos eventos que entran en la agenda por criterios de temporalidad, es decir, por la urgencia en ser difundidas, tanto como a la importancia política y cultural del tema que incluyen (Boczkowski, 2009: 100). Si además se agrega la dimensión narrativa, puede comprobarse que temas habitualmente considerados duros, como las campañas políticas, pueden ser presentados en formatos de infoentretenimiento, con recursos sensacionalistas o de personalización. Con esa complejidad resulta difícil tipificar los temas con mayor tratamiento de periodistas mujeres en los diarios analizados en las categorías de noticias duras o blandas, y este es un concepto tomado de la teoría del periodismo descuidado en los estudios de género que lo mencionan.

La constatación del bajo porcentaje de mujeres como protagonistas de la noticia llevó a preguntarse por la relación que existe entre la autoría y la representación de las mujeres en las noticias. El cuarto estudio GMMP de 2010 concluyó que había más mujeres en las noticias realizadas por las periodistas mujeres (28\%) que en los reportajes informados por los periodistas hombres (22\%) (World Association for Christian Communication, 2015). 
El estudio Journalistic Role Performance codificó las fuentes por tipo (Estado y partidos políticos, empresarios, sociedad civil, ciudadano común, medios, expertos, otros), considerando la presencia en la noticia como una, independientemente de las veces que apareciera. Como otra variable, se identificó cuál de todas las presentes era la fuente principal. Los resultados preliminares señalan que no se observan grandes diferencias, excepto que parece haber un mayor esfuerzo en ofrecer fuentes entre las mujeres. En las notas firmadas por hombres las notas sin fuentes o con una sola representan un tercio $(29,9 \%)$ mientras que en las firmadas por mujeres ese porcentaje baja a $18,6 \%$, pero los porcentajes se equilibran en las notas con más fuentes.

Investigaciones previas plantean una relación directa entre la proporción de mujeres en las redacciones y el tono y el estilo en el que se ofrecen las informaciones (Ross, 2001), argumentando que las mujeres enfocan sus noticias desde una perspectiva distinta y tienden a la elección de fuentes de información alternativas (Ross y Carter, 2016). Otras investigadoras concluyen que para que eso ocurra la influencia debe ejercerse desde posiciones directivas desde la que pueden asignar a mujeres los temas más importantes, como política, que en redacciones con predominio de hombre se asignan a colegas del mismo género (Craft y Wanta, 2004). Estas conclusiones plantean una pregunta derivada de la segunda hipótesis a resolver, que es si las mujeres tienen prácticas diferentes a las que tienen los hombres, que permitan pensar en un periodismo diferente, y si consultan mayor diversidad de fuentes o les habilitan voces distintas a las dominantes.

Un aspecto que merecería un estudio más detallado es el de las fuentes con las que tienen contacto. Los datos de Journalistic Role Performance muestran que tanto periodistas hombres como mujeres consultan el mismo tipo de fuente y coinciden en que su fuente principal es el Estado y la política. En la misma proporción consultan fuentes de la sociedad civil, que aparecen como fuente principal en una de cada diez notas, cualquiera sea el autor (mujer, hombre o no lleve firma). Las mujeres muestran una mejor predisposición a consultar personas corrientes como fuentes (16,5\% de las notas firmadas por ellas tienen ciudadanos como fuente principal) y a expertos $(15,2 \%)$; pero se trata de una tendencia y no de una diferencia sustancial con los colegas masculinos, que le conceden un $10,8 \%$ de sus notas a la ciudadanía como fuente principal y $8,9 \%$ para los expertos.

\section{Discusión: La palabra periodista pocas veces se refiere a una mujer}

Los datos empíricos que configuran el perfil de la periodista argentina confirman parcialmente las dos hipótesis planteadas al inicio. Por un lado, las periodistas argentinas tienen una participación minoritaria en los medios argentinos, pero no puede decirse que en todos ocurre de la misma manera. Los diarios parecen contar con plantas más igualitarias que otros medios como la televisión, aunque un análisis de contenido de sus noticias muestra una mayor 
participación de hombres en la firma de las notas. Este recurso otorga visibilidad del nombre propio y contribuye al reconocimiento del periodismo de mujeres, pero presenta una gran diferencia entre los cuatro diarios analizados que no puede explicarse con la posición editorial o ideológica del medio. Por caso un diario de elite de derecha es el que más firmas de mujeres en más temas más variados ofrece, mientras que el diario de elite de izquierda, identificado expresamente con las posiciones feministas muestra un desequilibrio en la asignación de temas y en la visibilidad de las firmas de mujeres. Lo propio pasa con los diarios populares, uno de los cuales muestra una mayor participación que el otro, que directamente no tiene ninguna firma de mujeres.

Esta conclusión relativiza la segunda parte de la hipótesis que hablaba de las posiciones subalternas de las periodistas, dado que varían mucho no solo entre tipo de medios, sino dentro de distintos medios dentro de un mismo tipo. La misma condición debe asociarse a la segunda hipótesis que sostenía que las periodistas argentinas se ocupan de temas accesorios a la agenda noticiosa de las secciones centrales de los periódicos. Los resultados muestran variaciones en los distintos medios, lo que plantea la necesidad de sumar información de contexto a la hora de analizar los resultados de las investigaciones sobre periodismo desde una perspectiva de género.

La televisión, medio que ha dominado en los estudios de género y ha sido la preocupación principal de las políticas públicas, puede no ser el medio más receptivo a las periodistas. Pero no puede obviarse que la TV tampoco es abierta al periodismo profesional en general, que en el conjunto de la programación es un género minoritario en comparación con el entretenimiento, la ficción y la publicidad. Justamente por mostrar un comportamiento distinto al del resto, puede discutirse si resulta un medio que pueda aportar referencias para la condición del periodismo en general y de las periodistas argentinas en particular. Más allá de su penetración en las audiencias, una cuestión son los contenidos y sus potenciales efectos en las audiencias y otra es el periodismo y los espacios informativos, cada vez más acotados en la televisión, que a veces se confunden en programas de variedades o talk shows, que invitan a sacar conclusiones alrededor de figuras televisivas que nada tienen que ver con el periodismo.

Las conclusiones dejan planteadas preguntas sobre los factores que pueden promover la participación de las mujeres en las redacciones. El periodismo es una profesión en crisis que está redefiniendo lo que se suponía era ser periodista (Mancini, 2013; Waisbord, 2013). Para discutir la identidad de las mujeres en la profesión es necesario revisar los enfoques con los que se la aborda: si como un servicio público de producción y distribución de la información, o una profesión definida por procedimientos como la objetividad, el reporte de la actualidad, o las pautas éticas; o si se define por su autonomía de otros actores sociales, la tecnología de la que se vale, o una visión de multiculturalismo (Deuze, 2005). Cada una de estas perspectivas es en sí misma una línea de estudios dentro del periodismo, con alcances y modelos muy diferentes, que incluyen y excluyen determinadas identidades profesionales.

El periodismo en general está discutiendo las exigencias de la profesión (horarios pautados por la naturaleza de los eventos, jornadas laborales extendidas), las nuevas formas de contratación 
(pérdida de exclusividad contractual, contratos free lance con más libertad, pero menos protección), las nuevas competencias técnicas (periodista multimedia, multifunción, multitarget). Estas cuestiones generales afectan al grupo profesional de las mujeres de particular manera (Ross, 2001), especialmente porque es una profesión donde hay un muy rápido y barato recambio para las posiciones inferiores y muchas exigencias para quienes logran una permanencia o la construcción de una marca con su nombre lo suficientemente fuerte para basar en ello su autonomía. También plantea preguntas sobre si hay una exclusión abierta de las mujeres en las redacciones o son las propias condiciones de trabajo de jornadas extendidas y traslados imprevistos las que la hacen una profesión expulsiva o poco atractiva para ellas. La escala salarial de la Tabla 4. muestra que los bajos salarios son un problema en general del periodismo, que en los puestos de rango inferior suele aceptar sueldos bajos o trabajar sin paga como condición naturalizada de ingreso a la profesión. La internalización de las pautas de trabajo lleva no solo a las y los periodistas a aceptar condiciones de trabajo inadmisibles en otras profesiones, con lo que se hace más delicado evaluar cuáles recaen especialmente en un género en particular. Esta naturalización explica por qué muchas entrevistas con profesionales no ofrecen los resultados que espera el observador, como señalaba Ross (2001) que echaba en falta entre las periodistas investigadas consensos alrededor de las cuestiones de género.

La reflexión de la pseudofeminización como efecto colateral de la participación de las mujeres en los medios complejiza aún más el estudio del tema (Soriano et al., 2005). Por caso, la mayor inserción de mujeres en el periodismo español se caracteriza por una alta tasa de abandonos, con puestos de escasa responsabilidad, mayor presencia en sectores considerados periféricos y trayectorias profesionales marcadas por desplazamientos hacia espacios profesionales menos visibles, pero más compatibles con la vida privada (Soriano, 2005: 366). Algo similar se observa con la formación universitaria de las mujeres, que no es garantía de acceso ni siquiera en países con políticas de cuotas participativas como Estados Unidos.

Esta tensión entre condiciones reales y condiciones ideales explica la paradoja de que el diario reconocido por su perspectiva de género, con periodistas que se identifican como feministas, sea el que menos visibilidad jerarquizada tienen en las noticias del cuerpo principal. Lo que a su vez cuestiona la presunción de que la visibilización de la perspectiva de género, o políticas públicas para la radio y la televisión, podrían garantizar igualdad de géneros en los medios informativos en general (Hassan y Gil, 2014). El porcentaje de la desigualdad de las mujeres con relación a sus colegas hombres no devela por sí solo las causas de los desequilibrios en una profesión con particularidades como el periodismo. Pero plantea la utilidad de partir de datos de campo para cuestionar el sentido común que construye el imaginario de cada medio y de cada periodista. Las entrevistas como instrumento de estudio aportan las percepciones de los individuos, pero necesitan datos para una triangulación que conforme un panorama más abarcador.

Los resultados del GMMP de 2015, pesimistas con relación a los avances de la mujer en el periodismo basándose en la baja participación en las noticias deben ponerse en un contexto 
nacional y regional para apreciar con perspectiva los cambios que muestran algunos países e indagar más en los factores que los propiciaron. En esta mirada diacrónica, son invalorables los registros sistemáticos de las condiciones laborales y profesionales del periodismo.

Sin perder la expectativa de que la progresiva incorporación de mujeres a la profesión transformará profundamente la estructura socio-demográfica del periodismo, la participación igualitaria en cualquier campo profesional es un tema de derechos laborales y humanos, que va más allá de las transformaciones que pueda traer en los contenidos periodísticos. Y estos son aspectos que merecen indagarse con más profundidad, entendiendo que la presencia de la mujer en los contenidos, en las aulas o en las redacciones son cuestiones con una fuerte interrelación, que no pueden analizarse separadamente de las fuerzas que atraviesan el campo profesional en el que acontecen.

\section{BIBLIOGRAFÍA}

- Altés, Elvira (2006): "La situación de la mujer en el proyecto global de monitoreo de medios". En: Actas del Foro Internacional de Comunicación e xénero, Pontevedra, pp. 473-478. Disponible en: https://dialnet.unirioja.es/descarga/articulo/2417003.pdf [25/02/2017].

- Amado, Adriana (2016): “Journalists in Argentina”. En: Worlds of Journalism Study-LudwigMaximilian Universität München. Disponible en: https://epub.ub.unimuenchen.de/29696/1/Adriana_Amado_Country_report_Argentina.pdf [17/02/2017].

- Amado, Adriana et al. (2016): "Periodismos latinoamericanos: perfil y roles profesionales". En: Adriana Amado y Martín Oller Alonso (eds.): El periodismo por los periodistas. Montevideo: Konrad Adenauer, Infociudadana, pp. 11-25

- Amado, Adriana y Pizzolo, Natalia (2014): “Journalism Studies in Argentina: Background y Questions". En: Brazilian Journalism Research, vol. 10, nº. 1, pp. 8-23.

- Amado, Adriana y Waisbord, Silvio (2015): "Divided We Stand: Blurred Boundaries in Argentine Journalism". En: Mathew Carlson y Seth Lewis (eds.): Boundaries of Journalism: Professionalism, Practices, and Participation. Nueva York: Routledge, pp. 51-66.

- Arrueta, César (2010): ¿Qué realidad construyen los diarios? Buenos Aires: La Crujía.

- Boczkowski, Pablo (2009): "Rethinking Hard and Soft News Production: From Common Ground to Divergent Paths". En: Journal of Communication, vol. 59, n. 1, pp. 98-116.

. (2010): News at Work: Imitation in an Age of Information Abundance. Chicago: University Of Chicago Press.

- Bourdieu, Pierre (2000): La dominación masculina. Barcelona: Anagrama.

- Chaer, Sandra y Santoro, Sonia (2007) (eds.): Las palabras tienen sexo: introducción a un periodismo con perspectiva de género. Buenos Aires: Artemisa.

- Chaer, Sandra (2007): "Medios masivos, medios alternativos y redes de periodistas". En: Sandra Chaer y Santoro, Sonia (eds.): Las palabras tienen sexo. Buenos Aires: Artemisa, pp. 
$111-124$.

- Craft, Stephanie y Wanta, Wayne (2004): "Women in the Newsroom: Influences of Female Editors and Reporters on the News Agenda". En: Journalism \& Mass Communication Quarterly; vol. $81, \mathrm{n}^{\circ} .1$, pp. 124-138.

- Dessein, Daniel y Roitberg, Gastón (2014): Nuevos desafíos del periodismo. Buenos Aires: Ariel.

- Deuze, Mark (2005): "What Is Journalism?: Professional Identity and Ideology of Journalists Reconsidered”. En: Journalism, vol. 6, n. 4, pp. 442-464.

- Ehrick, Christine (2016): Radio an the Gendered Soundscape. Women and Broadcasting in Argentina and Uruguay. Cambridge University Press.

- ENACON - Ente Nacional de Comunicaciones (n.d.): "Observatorio de la Discriminación en Radio y Televisión”. Disponible en: http://www.obserdiscriminacion.gob.ar [19/02/2017].

- Femenías, María Luisa. (2000): Sobre sujeto y género. Buenos Aires: Catálogos.

- Foro de Periodismo Argentino (2005): “Sobre los periodistas y su profesión”. Buenos Aires: Fopea. Disponible en: http://www.fopea.org/Etica/Encuesta_sobre_Periodismo [25/02/2017].

. (2011): Encuesta sobre los periodistas y su profesión. Buenos Aires. Disponible en: http://www.www.fopea.org/Recursos/Biblioteca_Virtual/Estudios/Clima_de_la_Actividad_Peri odistica_de_la_Argentina [25/02/2017].

. (2014): "Clima de la actividad periodística en Argentina". Buenos Aires. Disponible en: http://www.fopea.org/Inicio/Encuesta_sobre_la_autocensura_en_el_periodismo_argentino [25/02/2017].

- Gobbi, Maria Cristina y Betti, Juliana (2013): "Formação E Cognição: Ensino Da Comunicação No Brasil E No Cone Sul”. En: José Marques de Melo y João Cláudio Garcia Rodrigues Lima (eds.): Panorama da comunicação e das telecomunicações no Brasil, San Pablo: Ipea Instituto de Pesquisa Econômica Aplicada. pp. 13-163.

- Hassan, Valeria y Gil, Soledad (2014): "Estrategias del periodismo feminista: prácticas y política en la reconfiguración del espacio comunicacional". En: Perspectivas de la Comunicación, vol. 7, pp. 84-96.

- Humanes, María Luisa (2003): "Evolución de roles y actitudes . cultura y modelos profesionales del periodismo". En: Telos. Cuadernos de Comunicación, Tecnología y Sociedad, vol. 54, abril, pp. 1-10.

- Journalistic Role Performance around the Globe (n.d.): "The Concept of Journalistic Role Performance". Disponible en: http://www.journalisticperformance.org/p/the-project.html [10/02/2017].

- Lamas, Marta (1999): “Usos, dificultades y posibilidades de la categoría género". En: Papeles de Población, vol. 5, nº. 21, pp.147-178.

- Laudano, Claudia (2010): "Mujeres y medios de comunicación: reflexiones feministas en torno a diferentes paradigmas de investigación.” En: Sandra Chaer y Sonia Santoro (eds.): Las palabras tienen sexo. Buenos Aires: Artemisa, pp. 40-54. 
- Luchessi, Lila y Videla, Luciano (2016) (eds.): Desafíos del periodismo en la sociedad del conocimiento. Viedma: Universidad Nacional de Río Negro.

- Mancini, Paolo (2013): "What Scholars Can Learn from the Crisis of Journalism". En: International Journal of Communication, vol. 7, pp. 127-136.

- Martini, Stella y Luchessi, Lila (2004): Los que hacen la noticia. Buenos Aires: Biblos.

- Mellado, Claudia (2015): "Professional Roles in News Contents: Six Dimensions of Journalistic Role Performance”. En: Journalism Studies, vol. 16, nº 4, pp. 596-614.

- Ministerio de Educación de la Argentina (2014): "Síntesis de Información Estadísticas Universitarias Argentina 2014-2015". Departamento de Información Universitaria. Disponible en:

http://portales.educacion.gov.ar/spu/wpcontent/blogs.dir/17/files/2015/12/Sintesis_completa.pdf [26/02/2017].

- Journalistic Role Performance around the Globe (n.d): "The Concept of Journalistic Role Performance". Disponible en: http://www.journalisticperformance.org/p/the-project.html [10/02/2017].

- Ministerio de Educación de la Argentina (2011): “Anuario 2011. Estadísticas Universitarias”. Disponible en: http://informacionpresupuestaria.siu.edu.ar/DocumentosSPU/Anuario\%20de\%20Estad\%C3\%A Dsticas\%20Universitarias\%20-\%20Argentina\%202011.pdf [26/02/2017].

- Mitchelstein, Eugenia y Pablo Boczkowski (2009): “Between Tradition and Change.” En: Journalism, vol. 10, $\mathrm{n}^{\circ} .5$, pp. 562-586.

- North, Louise (2016): “The Gender of 'soft' and 'hard' News”. En: Journalism Studies, vol. $17, \mathrm{n}^{\mathrm{o}} .3$, pp. 356-373.

- Rivero Santamarina, Diana; Meso Ayerdi, Koldo y Peña Fernández, Simón (2015): "La feminización de los estudios de periodismo: análisis del caso español”. En: Revista Latina de Comunicacion Social, vol. 70, pp. 566-583.

- Rivero Santamarina, Diana (2014): "División sexual del periodismo: ellas en las aulas y ellos en las redacciones.”. En: Cuestiones de género: de la igualdad y la diferencia, n. 9, pp. 259282.

- Ross, Karen (2001): “Women at Work: Journalism as En-Gendered Practice”. En: Journalism Studies, vol. 2, no. 4, pp. 531-544.

- Ross, Karen; Boyle, Karen; Carter, Cynthia y Ging, Debbie (2016): “Women, Men and News". En: Journalism Studies, vol. 9699, September, pp. 1-22.

- Ross, Karen y Carter, Cynthia (2016): "Women and News: A Long and Winding Road". En: Media Culture \& Society, vol. 33, nº. 8, pp. 1148-1165.

- Rost, Alejandro y Liuzzi, Álvaro (2012): "Reorganización de las redacciones y nuevos perfiles profesionales". En: Alejandro Rost y Fabián Bergero (eds.): Periodismo en contexto de convergencias. General Roca: Publifadecs, pp. 13-52.

- Rovetto, Florencia (2012): “Estudios feministas y medios de comunicación: avances teóricos 
y periodísticos en España y Argentina”. En: Revista F@ ro, vol. 1, nº 16, pp. 14-27.

. (2013): "Percepciones sobre desigualdades de género en el trabajo periodístico". En: Global Media Journal México, vol. 10, n. 20, pp. 54-73.

- Santoro, Sonia y Chaer, Sandra (2010) (eds.): Las palabras tienen sexo. Herramientas para un periodismo de genero. Buenos Aires: Artemisa.

- Scott, Joan W. (1996): "El género: una categoría útil para el análisis histórico". En: Marta Lamas (ed.): El género: la construcción cultural de la diferencia sexual. México: PUEG, pp. 265-302.

- Shoemaker, Pamela y Reese, Stephen (1996): Mediating the Message. Theories of Influences on Mass Media Content. 2nd. Nueva York: Longman.

- Soriano, Jaume; Cantón, María y Díez, Mercè (2005): “La pseudofeminización de la profesión periodística en españa”. En: Zer - Revista de Estudios de Comunicación, vol. 10, nº 19, pp. 3552.

- Tuchman, Gaye (1978): Making News. A Study in the Construction of Reality. New York: The Free Press.

- Valles Ruiz, Rosa María (2006): "Mujeres periodistas: empoderamiento restringido". En: Revista Mexicana de Ciencias Políticas y Sociales, vol. 48, nº 197, pp. 137-147.

- Waisbord, Silvio (2013): Reinventing Professionalism. Cambridge: Polity Press.

- Waisbord, Silvio y Amado, Adriana (2014): "Periodismo partido al medio". En: Adriana Amado (ed.): La comunicación pública como espectáculo. Buenos Aires: Konrad Adenauer, pp. 211-285.

- Weaver, David (1999): "Periodismo y nuevas Tecnologías : Perfiles de los periodistas del siglo XXI”. En: CIC Cuadernos de información y comunicación, vol. 4, pp. 219-230.

- Weaver, David y Willnat, Lars (2012): The Global Journalist in the 21st Century. Nueva York: Routledge.

- West, Candice; Lazar, Michele y Chramare, Cheris (2000): "El género en el discurso". En: TeunVan Dijk (ed.): El discurso como interacción social: estudios del discurso, introducción multidisciplinaria., Barcelona: Gedisa, pp. 179-212.

- World Association for Christian Communication (2015): "Monitoreo Global de Medios. Resumen". Disponible en: http://whomakesthenews.org/gmmp-2015 [26/02/2017]

- WJS - Worlds of Journalism Study (2016): “The WJS 2012-2016 Study. Methodological Documentation". Disponible en: http://www.worldsofjournalism.org/research/2012-2016study/methodological-documentation/ [25/02/2017].

- Worlds of Journalism (2016): “Aggregated tables for key variables (2012-2016): Sociodemographic backgrounds". Disponible en: http://www.worldsofjournalism.org/fileadmin/Data_tables_documentation/Tables/WJS_Sociod emographic_backgrounds_-_aggregated.pdf [25/2/2017]. 